\title{
Compromisos epistémicos en el enfoque estructuralista de las teorías*
}

\section{(Epistemic Commitment in Structuralist Approach of Theories)}

\author{
Germán Guerrero Pino
}

Recibido: 14 de septiembre de 2011

Aceptado: 20 de diciembre de 2011

\section{Resumen}

Una idea ampliamente extendida es que el programa estructuralista es completamente neutral en cuestiones epistémicas. Por el contrario, el objetivo del artículo es mostrar que el estructuralismo es epistémicamente neutral en el debate realismo vs. antirrealismo, pero que supone un compromiso epistémico en la fundamentación de las teorías. Este compromiso epistémico tiene las características de un coherentismo; y dos tesis estructuralistas conducen a ello: que una teoría se contrasta empíricamente a través de sus aplicaciones propuestas y que las aplicaciones propuestas de una teoría quedan descritas únicamente en función de conceptos que son no-teóricos respecto a ésta teoría.

Palabras clave: Estructuralismo, teoría, fundamentismo, coherentismo, experiencia, van Fraassen.

\begin{abstract}
The idea that the Structuralist Program is completely neutral in epistemological matters is widely extended. On the contrary, the article's purpose is to show that

\footnotetext{
* Si bien las ideas centrales de este trabajo se encuentran en Guerrero (2003), aquí se presentan y sustentan de manera más sistemática y con argumentos adicionales, los cuales presenté en el XV Congreso Internacional de Filosofía, Asociación Filosófica de México, Ciudad de México, del 25 al 29 de enero de 2010, en donde tuve la oportunidad de recibir comentarios críticos de Mario Casanueva y Pablo Lorenzano (a quienes agradezco) que me ayudaron a mejorar algunas de las ideas aquí expuestas. Igualmente agradezco a uno de los informadores anónimos de la Revista de Filosofía, sus comentarios y sugerencias a una versión anterior de este trabajo.
} 
while it is true that structuralism is neutral in the epistemological debate between realism and antirealism, structuralism supposes an epistemic commitment in the foundation of theories. This epistemic commitment has the characteristics of a coherentism and two structuralist thesis lead precisely to that: scientific theories are contrasted through its intended applications and the intended applications of a theory are described only in non- theoretical concepts relative to theory.

Keywords: Structuralism, theory, foundationalism, coherentism, experience, van Fraassen.

\section{Introducción}

Una idea ampliamente extendida entre partidarios y críticos de la concepción estructuralista de las teorías científicas es que ésta es completamente neutral en cuestiones epistémicas. La neutralidad epistemológica, en el contexto del enfoque semántico de las teorías, es acuciante especialmente en dos cuestiones de interés: el debate realismo vs. antirrealismo y la evaluación de las teorías, que tiene que ver con la forma como se caracteriza la base empírica de contrastación de las teorías ${ }^{1}$.

El objetivo de este artículo es mostrar que el programa estructuralista de las teorías es epistémicamente neutral en el debate realismo vs. antirrealismo, pero que supone un compromiso epistémico en la evaluación de las teorías. Este compromiso epistémico tiene las características de un coherentismo. Considero que dos tesis estructuralistas son las que lo llevan al coherentismo: una, que una teoría se contrasta empíricamente a través de sus aplicaciones propuestas y, dos, que las aplicaciones propuestas de una teoría quedan descritas únicamente en función de términos que son no-teóricos respecto a la teoría en consideración.

Para ello, se comienza por presentar los elementos más sustantivos del programa estructuralista que permitan enunciar, precisar y sustentar las ideas y argumentos que se van a desarrollar con miras al objetivo central. No es fácil hacer esto en pocas páginas, sin obviamente desdibujar el espíritu general del programa estructuralista, así que espero lograr un equilibrio entre la extensión de dicha exposición y la claridad y precisión en las ideas centrales del programa estructuralista. Esto se

\footnotetext{
${ }^{1}$ El debate realismo e instrumentalismo también puede plantearse respecto al progreso científico y, en particular, dentro de la concepción estructuralista de las teorías, tal y como ha sido discutido, por ejemplo, por Rivadulla (1985). La tesis central de Rivadulla (1985, p. 254) es que "la descripción que el estructuralismo hace del desarrollo de la ciencia es susceptible de recibir una interpretación realista que, además, explica el éxito pragmático de la ciencia”. Esto mostraría que el estructuralismo asume un compromiso epistémico en cuanto al progreso. Ahora bien, este debate no será objeto de análisis en este trabajo, aunque tiene relación con el tema de la evaluación de las teorías, porque desborda sus límites.
} 
hace en el segundo apartado. En el tercer apartado se justifica la idea de la neutralidad del estructuralismo en la discusión sobre el realismo científico estableciendo un paralelo con la forma que adquiere el antirrealismo de van Fraassen dentro de su particular versión del enfoque semántico. En el último apartado, en primer lugar, se desarrolla en forma amplia el argumento que muestra cómo los planteamientos estructuralistas desembocan en un coherentismo, siguiendo muy de cerca los argumentos presentados por Balzer, Moulines y Sneed (1987). En segundo lugar, se argumenta brevemente (por no ser el foco de atención de este escrito) por qué dicho coherentismo es inaceptable, y se presenta en forma esquemática una posible solución empirista (moderada) al tema de la justificación de la teorías, siguiendo una estrategia semejante a la anterior de contrastar los planteamientos estructuralistas con los de van Fraassen.

\section{La versión estructuralista del enfoque semántico}

El programa estructuralista de las teorías científicas es una de las versiones ${ }^{2}$ del enfoque semántico o modelo-teórico de las teorías, el cual surge como alternativa al enfoque sintáctico (o concepción heredada o enfoque sintáctico-axiomático o concepción enunciativa ${ }^{3}$ o enfoque lingüístico) de las teorías. De acuerdo con el enfoque sintáctico, una teoría es un conjunto de enunciados organizados deductiva o axiomáticamente, en sentido estricto. En términos más precisos, una teoría científica es un cálculo (o sistema) formal axiomatizado, parcialmente interpretado mediante reglas de correspondencia (o de interpretación) que relacionan términos teóricos con términos observacionales. A este enfoque se le califica de sintáctico porque todo cálculo formal se encuentra asociado con la sintaxis de un lenguaje formal y, además, la derivabilidad en un lenguaje formal es una noción sintáctica. A manera de ilustración, bajo esta perspectiva, la mecánica newtoniana equivale, básicamente, a sus leyes o principios (las tres leyes de movimiento más la ley de gravedad) más todo lo que de ellas se deduce.

En tanto que el enfoque semántico propone que una teoría queda mejor comprendida como un conjunto de modelos ${ }^{4}$, esto es, como el conjunto de estructuras

\footnotetext{
2 Estas son las distintas versiones del enfoque semántico: la estándar de P. Suppes; la de espacios de estados de C. B. van Fraassen, R. Giere y F. Suppe; la estructuralista de W. Stegmüller, J. Sneed, W. Balzer y C. U. Moulines; la de M. L. Dalla Chiara y G. Toraldo di Francia en Italia; la de M. Przełecki y R. Wójcicki en Polonia; y la del grupo de N. da Costa y S. French en Brasil.

${ }^{3}$ Estas expresiones fueron introducidas por Putnam (1962), van Fraassen (1970) y Stegmüller (1976), respectivamente.

${ }^{4}$ Los estructuralistas asumen el término modelo en el sentido de la semántica formal, esto es, como estructuras que satisfacen determinados axiomas, véase Moulines (2002), p. 5. En lo que sigue nos atendremos a esta interpretación, aunque no es importante en el presente contexto.
} 
caracterizadas o identificadas a través de las leyes o principios de la teoría. Debido a que este enfoque enfatiza los modelos y a que, en la semántica formal o teoría de modelos, la noción de modelo es una noción semántica, es que a este enfoque se le califica de semántico o modelo-teórico. Así, y como ilustración, de acuerdo con esta perspectiva, en el caso de la mecánica newtoniana el énfasis no hay que hacerlo en sus leyes sino en los modelos (o sistemas idealizados) que éstas (las leyes o principios) describen, caracterizan o definen; modelos como el siguiente: un conjunto de partículas puntuales con masa que interactúan entre sí mediante fuerzas de diversos tipos, de modo que estas interacciones se dan de acuerdo con las leyes de Newton.

La obra fundacional del programa estructuralista es The Logical Structure of Mathematical Physics (1971), de Joseph Sneed, y el libro An Architectonic for Science (1987), escrito por Balzer, Moulines y Sneed, se considera la obra madura del programa. Este programa ha hecho de la identidad, estructura, relaciones mutuas y evolución de las teorías científicas el foco de su investigación en el campo de la filosofía de la ciencia, de modo que ha logrado una caracterización bastante fina y completa de estas distintas dimensiones de una teoría empírica. Dicho esto, mi propósito a continuación no podrá ser exponer el programa estructuralista en sus distintos detalles, sino que sólo me ocuparé de presentar los aspectos sustantivos de la reconstrucción metateórica de las teorías científicas propuesta por el programa estructuralista que me permitan precisar aquellas nociones y tesis relacionadas con el objetivo del presente escrito, sin desdibujar el espíritu general de dicho programa. Me detendré en la noción más simple de teoría científica que han propuesto los estructuralistas y en la caracterización de sus principales elementos 5 .

En cuanto a lo primero, de acuerdo con los estructuralistas, la unidad más simple que se puede considerar como una teoría científica es la estructura $T=\langle K, I\rangle$, en donde $K$ es el núcleo formal e $I$ el dominio de aplicaciones propuestas del elemento teórico $T$ (o teoría, para el presente estudio) ${ }^{6}$. El núcleo (teórico) $K$ es la parte formal de $T$ y contiene los diferentes elementos conceptuales que permiten caracterizar el dominio de estudio y las supuestas leyes que lo rigen. El campo de aplicaciones propuestas $I$ es, como su nombre lo indica, la parte aplicativa de $T$ y contiene los sistemas reales, empíricos, a los cuales se pretende aplicar las leyes de $K$. En los términos del ejemplo de arriba referido a la mecánica newtoniana, tenemos que, por una parte, el núcleo está relacionado con la definición o caracterización de los modelos a través de las conocidas leyes de Newton de la mecánica y, hablando estrictamente, equivale al conjunto de sistemas mecánicos newtonianos determinados por dicha definición. Por otra parte, nuestro sistema solar y cada uno de los sistemas Tierra-cuerpo (que ilustran la caída de los cuerpos) son, en sentido estricto,

\footnotetext{
5 En Balzer y Moulines (2000) se encuentra una buena síntesis de las ideas centrales del programa. En español, se puede consultar Díez y Moulines (1997).

6 Véase Balzer y Moulines (2000), p. 17.
} 
aplicaciones propuestas de la mecánica newtoniana, es decir, cada uno de estos sistemas pertenece al conjunto $I$.

Más en detalle, el núcleo formal $K$ tiene como componentes a $M_{p}$ (modelos potenciales), $M$ (modelos actuales), $G C$ (condición de ligadura global -global constraint-), $G L$ (vínculo global - global link) y $M_{p p}$ (modelos parciales). De modo que podemos escribir $K=\left\langle M_{p}, M, M_{p p}, G C, G L\right\rangle$. En lo que sigue sólo presentaré, en términos amplios, las tres primeras componentes de $K$ y las aplicaciones propuestas, que son los puntos más pertinentes y relevantes para la discusión en este artículo.

Modelos potenciales $\left(M_{p}\right)$. Los estructuralistas adoptan como forma estándar la de definir los modelos de una teoría mediante un predicado conjuntista a través de lo que se conoce como axiomatización conjuntista informal. Un predicado conjuntista tiene la forma " $x$ es un modelo de la teoría .... syss $_{\text {def }} \varphi(x)$ ", donde $\varphi(x)$ expresa principalmente, aunque no exclusivamente, las leyes de la teoría. Aquí, $x$ es una estructura matemática y las distintas estructuras matemáticas seleccionadas mediante el predicado conjuntista son los modelos de la teoría. Además, cada elemento de $\varphi(x)$ establece restricciones a la estructura $x$ y a esos elementos se les conoce como cláusulas o axiomas del predicado conjuntista. En el conjunto de axiomas es posible distinguir dos tipos de axiomas, los impropios y los propios de la teoría. Los axiomas propios equivalen a lo que corrientemente se llama leyes o principios de la teoría, y los impropios establecen restricciones sólo de tipo lógico-matemático, proporcionando los diferentes elementos conceptuales globales de la teoría. Pues bien, un modelo potencial de una teoría es una estructura que satisface los axiomas impropios del correspondiente predicado conjuntista de la teoría y $M_{p}$ es el conjunto de todos los modelos potenciales.

Modelos actuales $(M)$. Un modelo actual es una estructura que satisface tanto los axiomas impropios como los propios del predicado conjuntista; así, un modelo actual es un modelo potencial que satisface los axiomas propios del predicado conjuntista, aunque no necesariamente todo modelo potencial es modelo actual. Por tanto, se cumple que $M \subseteq M_{p}$.

T-teoricidad. Con el propósito de caracterizar de manera apropiada la base empírica y la aserción empírica de una teoría es importante precisar el criterio de teoricidad relativa de los conceptos y, después, la noción de modelo parcial. Dada una teoría $T$ es posible distinguir dos tipos de conceptos en su interior, los que son propios de la teoría $T$ y los que no lo son; o, en otros términos, los que son directamente introducidos por $T$ y aquellos con los cuales se contaba con anterioridad a $T$. Los primeros son teóricos respecto a $T$, esto es, son $T$-teóricos y dependen por completo de T; y los segundos son no-teóricos respecto a $T$, esto es, son $T$-no-teóricos y no dependen estrictamente de $T$, aunque han de ser teóricos respecto a otra teoría diferente. En general, todo concepto es teórico, es introducido por una teoría particular, pero respecto a una teoría $T$ podemos hablar de conceptos $T$-teóricos y con- 
ceptos $T$-no-teóricos. Así, el carácter teórico (o no-teórico) de un concepto no está dado en términos absolutos sino en relación con una teoría ${ }^{7}$. De manera más precisa, un concepto será $T$-teórico si para su determinación hay que hacer uso de las leyes de $T$; mientras que si el concepto es $T$-no-teórico habrá procedimientos de determinación del concepto en los que no se empleen las leyes de $T$, muy seguramente habrá que emplear leyes de teorías anteriores a $T^{8}$.

Veamos un ejemplo. Los principales conceptos contenidos en la mecánica clásica $(M C)$ son posición, tiempo, velocidad, aceleración, masa y fuerza. Si nos acogemos a su desarrollo histórico, parece evidente que los conceptos de masa y fuerza fueron introducidos por primera vez en la física por Newton, en tanto que los conceptos fundamentales de espacio y tiempo, y con ellos los conceptos derivados de velocidad y aceleración, ya habían sido introducidos y precisados con anterioridad por la cinemática clásica $(C C)$ elaborada por Galileo, principalmente, y otros. Así, tenemos que posición, tiempo, velocidad y aceleración son conceptos $M C$-noteóricos y $C C$-teóricos, y que masa y fuerza son $M C$-teóricos.

Modelos parciales $\left(M_{p p}\right)$. Los modelos potenciales parciales o, simplemente, modelos parciales se obtienen de los modelos potenciales pero sin considerar (recortándoles) los conceptos $T$-teóricos. En el ejemplo anterior de la $M C$, los modelos potenciales de la $M C$ expresan todos los conceptos de la $M C$ (posición, tiempo, velocidad, aceleración, masa y fuerza) en términos lógico-matemáticos sin tener en cuenta las relaciones entre estos conceptos dadas por las leyes, en tanto que los modelos parciales sólo expresan los conceptos cinemáticos (posición, tiempo, velocidad y aceleración), los conceptos $M C$-no-teóricos. Por ejemplo, la caída de un cuerpo sobre la Tierra se puede describir con sólo conceptos cinemáticos diciendo que el cuerpo cae con cierta aceleración, lo cual sería un modelo parcial; pero también se puede describir dinámicamente diciendo que el cuerpo cae con cierta aceleración debido a la atracción gravitacional que ejerce la Tierra, lo cual sería un modelo potencial. El conjunto de modelos parciales de una teoría se representa por $M_{p p}$ y se puede determinar de manera precisa mediante las herramientas de la teoría de conjuntos.

Aplicaciones propuestas (I). Una de las principales novedades del programa estructuralista respecto a la versión estándar del enfoque semántico, en la que una teoría equivale estrictamente a un conjunto de modelos, es la de introducir las aplicaciones propuestas o pretendidas en la identidad de una teoría. Podemos entender

\footnotetext{
${ }^{7}$ La dicotomía T-no-teórico/T-teórico es diferente de la dicotomía teórico/observacional de los positivistas lógicos. La última dicotomía es imprecisa porque en realidad encierra dos pares de distinciones: la observable/inobservable, que se aplica a entidades, propiedades, eventos y procesos; y la $T$-no-teórico/ $T$-teórico, que se aplica a términos o conceptos.

8 Para el criterio preciso de teoricidad y el concepto de determinación remito a Balzer, Moulines y Sneed (1987), p. 55.
} 
las aplicaciones propuestas, o el dominio de aplicación de una teoría, como el conjunto formado por los distintos fenómenos naturales (o sistemas reales) a los cuales se pretende aplicar la teoría o que pretende explicar la teoría. Una ilustración, los sistemas Luna-Tierra, Tierra-Sol y un columpio en movimiento son fenómenos naturales que hacen parte de las aplicaciones propuestas de la mecánica newtoniana.

Dos son las principales razones que aducen los estructuralistas para que $I$ haga parte de una teoría empírica: porque aseguran que se trata de una teoría empírica y no de otra clase de teoría, por ejemplo de una teoría matemática; y porque permiten recuperar las actitudes doxásticas que los científicos tienen hacia las teorías. Si nos quedamos con sólo el núcleo $K$ en la identidad de una teoría, no es posible explicitar si la teoría es verdadera o falsa, adecuada o inadecuada, correcta o incorrecta, porque tales predicados se aplican a enunciados, a entidades lingüísticas, y los modelos no son entidades lingüísticas. Una vez se cuenta con $I$ como parte de una teoría, éste se puede relacionar con el núcleo $K$ a través de las aserciones empíricas que son enunciados y que, como tales, pueden ser verdaderas o falsas.

Una característica importante de las aplicaciones propuestas es que se describen a través de conceptos $T$-no-teóricos. Una aplicación no está dada independientemente de una conceptualización, sino que su descripción se debe dar mediante un aparato conceptual. De manera más concreta, los fenómenos pertinentes para una teoría particular $T$ no pueden estar conceptualizados de cualquier forma sino que deben estarlo en términos de un aparato conceptual compatible con $T$; pero éste no puede ser todo el aparato conceptual global de $T$ sino el que sólo incluya los conceptos $T$-no-teóricos, es decir, "lo que es dado para una teoría son los hechos establecidos por otras teorías" (Balzer, Moulines y Sneed, 1987, p. 86). En otras palabras, los sistemas reales deben estar descritos en términos del aparato conceptual proporcionado por $M_{p p}$ y no por el proporcionado por $M_{p}$, lo cual significa que toda aplicación propuesta es un modelo parcial o, lo que es lo mismo, que $I \subseteq M_{p p}$. He aquí la importancia de haber definido con anterioridad los modelos parciales.

¿Por qué los sistemas empíricos relevantes tienen que identificarse únicamente mediante términos no-teóricos, de acuerdo con los estructuralistas?; en otras palabras, ¿qué problema habría en identificarlos mediante parámetros teóricos u otros? De acuerdo con los estructuralistas, por una parte, hay que tener presente que la contrastación de la teoría con la experiencia se hace en concreto a través de las aplicaciones propuestas, de tal manera que si las aplicaciones propuestas están descritas en términos de la teoría mediante conceptos $T$-teóricos, entonces éstas presupondrían la teoría y se caería así en un círculo vicioso: pues las experiencias que permiten contrastar la teoría están descritas en términos de la misma teoría. Esta situación sería tanto como admitir que la teoría es a priori válida. Por otra parte, tampoco pueden estar descritas las aplicaciones propuestas mediante un aparato conceptual extraño por completo a la teoría, pues de ser así no se podría establecer rela- 
ción alguna entre aplicaciones y teoría. Los conceptos no-teóricos superan estos dos escollos, no son extraños a la teoría y, al mismo tiempo, no la presuponen.

Esta tesis estructuralista, de que los fenómenos hay que describirlos mediante conceptos no-teóricos, es la pieza clave del análisis que se hará más adelante con el objeto de defender que el programa estructuralista cae en un coherentismo en la evaluación de las teorías, por tanto es necesario detenernos aquí un momento para dejar claro que efectivamente esta tesis es parte importante de ese programa, así afirmaciones por parte de algunos estructuralistas intenten debilitar dicho compromiso. Muestra de esto último es la siguiente afirmación de Balzer y Moulines (2000, p. 16) respecto a que hay dos maneras posibles de caracterizar las aplicaciones propuestas: "la primera alternativa sería expresada mediante la fórmula $I \subseteq M_{p}$, mientras que la segunda mediante $I \subseteq M_{p p}$. La hipótesis normal del estructuralismo es que, al menos en muchas teorías, la alternativa más plausible es la última".

Pero, por ejemplo, en An Arquitectonic for Science es muy claro el compromiso con la tesis. Allí, desde un comienzo (véase página 40) se deja muy claro la importancia del tema para la filosofía de la ciencia y para el mismo programa estructuralista, y unas páginas más adelante se afirma "la base empírica de una teoría dada $T$ debería incluir aquellos conceptos que, en un sentido aún no precisado, no son específicos de la teoría" (p. 48). Y, después, dicha idea se precisa mucho más: "es más económico y natural asumir que las aplicaciones propuestas de $T$ tienen la estructura de los modelos potenciales parciales" (p. 86). Aún más, para no dejar dudas, finalmente se presenta la definición formal de aplicación propuesta, en donde una de las dos condiciones es "(2) $I \subseteq M_{p p}$ " (p. 88).

\section{Neutralidad epistémica del estructuralismo en el debate realismo/ antirrealismo}

Con miras a precisar la idea de que el programa estructuralista es neutral en el debate realismo vs. antirrealismo, una buena estrategia puede ser contrastarlo con la versión particular de van Fraassen del enfoque semántico de las teorías y con la forma que toma su empirismo constructivo dentro de ese enfoque.

Respecto a lo primero (la versión semántica particular de van Fraassen sobre las teorías), van Fraassen también considera insuficiente identificar una teoría empírica sólo con el conjunto de modelos, por lo menos es necesario incluir además un conjunto de hipótesis teóricas que relacionen los modelos con los sistemas empíricos. Con sus propias palabras: "presentar una teoría es definir un cierto tipo (o varios tipos) de sistemas más una o más hipótesis acerca de la relación de ciertos (tipos de) sistemas reales con la(s) clase(s) definida(s). Decimos, entonces, que la definición teórica y las hipótesis teóricas constituyen conjuntamente la formulación 
de la teoría dada" (van Fraassen, 1989, p. 226). La definición teórica se enuncia a través de las leyes o principios de la teoría y caracteriza la clase de modelos de la teoría, tal y como hemos visto arriba en el caso del programa estructuralista. En tanto que una hipótesis teórica afirma, en general, que algunos de los modelos tienen cierta relación (identidad, aproximación o subsunción) con los fenómenos observables, los sistemas empíricos determinados por los datos. Así, una hipótesis teórica postula una relación particular entre mundo y teoría (modelos).

Ahora bien, es necesario distinguir el enfoque semántico de las teorías de una postura realista o antirrealista de las teorías: lo primero tiene que ver con la naturaleza y estructura de las teorías, que son aspectos internos de una teoría, y lo segundo con la cuestión epistemológica de qué dice una teoría acerca del mundo natural, que es una cuestión sobre la relación teoría y mundo. De modo que la caracterización anterior de las hipótesis teóricas se ha hecho de manera general, sin proponer una relación específica entre modelos y fenómenos; no se dice si dicha relación es la de verdad (posición realista) o la de adecuación empírica (posición antirrealista) o cualquier otra. En síntesis, el enfoque semántico sobre la estructura de las teorías científicas es por completo neutral respecto al debate realismo/antirrealismo. De ahí que van Fraassen (Ibíd., p. 188. Lo del paréntesis es mío) afirme: “yo expongo el enfoque semántico en el contexto de mi antirrealismo [el empirismo constructivo], el cual otros pueden no compartir, pero el enfoque semántico no está ligado a éste".

Sobre la forma que toma el empirismo constructivo de van Fraassen, éste tiene que ver con el debate sobre la existencia o no de los objetos, propiedades y sucesos inobservables que son postulados por las teorías científicas para explicar los fenómenos observables. De acuerdo con el realismo científico hay buenas razones para pensar que los objetos y las propiedades inobservables que postula la ciencia realmente existen: las mismas teorías dicen que eso es así y esas teorías tienen un gran poder explicativo y predictivo. Por ejemplo, si una teoría física sobre la constitución de la materia está muy bien confirmada y ha permitido muchas explicaciones, entonces muy seguramente existen aquellos objetos y sus propiedades inobservables que propone, objetos tales como el electrón y propiedades como su carga. Mientras que el empirismo constructivo de van Fraassen (1980, p. 64. Lo de los paréntesis es mío) afirma que "presentar una teoría consiste en especificar una familia de estructuras, sus modelos [concepción semántica]; y, en segundo lugar [posición empirista], especificar ciertas partes de esos modelos (las subestructuras empíricas) como candidatos para la representación directa de los fenómenos observables". Tal y como van Fraassen plantea la cuestión, hay un punto o presupuesto que su empirismo constructivo comparte con los realistas, véase Rosenberg (2005, p. 97): la distinción observable/inobservable, esto es, que las teorías científicas buscan dar cuenta de los objetos observables, de sus propiedades observables y de sus relaciones observables mediante la postulación de objetos, propiedades y sucesos que 
son inobservables. Por tanto, la diferencia radica, más bien, en la forma como interpretan lo que las teorías dicen acerca del mundo.

Para el realista, lo que dicen las teorías científicas sobre el mundo no se restringe exclusivamente al ámbito de lo observable, ellas también dan cuenta de objetos, propiedades y sucesos inobservables, en la medida de su relativo éxito explicativo y predictivo. Así, para el realista el objetivo de la ciencia es proporcionar teorías verdaderas sobre cómo es el mundo, y dicha verdad trasciende el dominio de los fenómenos observables. En términos semánticos, diremos que una teoría es verdadera si entre la clase de sus modelos teóricos hay uno que se corresponde o es isomorfo con el mundo. Contrariamente, desde la perspectiva del empirismo constructivo de van Fraassen, la ciencia busca darnos teorías que sean verdaderas sólo en el ámbito de lo observable, esto es, teorías que salven los fenómenos (que de por sí son observables); en otras palabras, tal y como se subrayó arriba, teorías que sean empíricamente adecuadas, es decir (en términos semánticos), si entre los modelos de la teoría hay uno en el que cada fenómeno observable es isomorfo con alguna subestructura empírica de ese modelo. En definitiva, para van Fraassen, el realista se caracteriza por pensar que la verdad es el criterio de éxito de la ciencia y que aceptar una teoría es creer que es verdadera, en tanto que desde su empirismo constructivo el éxito de la ciencia radica en la adecuación empírica de las teorías y aceptar una teoría sólo nos compromete con la creencia en que sea empíricamente adecuada. Más allá de este dominio observacional, en el ámbito teórico de las entidades postuladas o inobservables, no tenemos nada garantizado, de modo que lo más prudente es suspender el juicio en relación con la existencia o no de dichas entidades. Ésa es la principal razón que esgrime van Fraassen (1980, p. 69) para asumir una filosofia empirista en vez de una realista, con sus propias palabras: "la afirmación de adecuación empírica es bastante más débil que la afirmación de verdad, y la resistencia a aceptar nos libera de la metafísica". Finalmente, para van Fraassen (Ibíd., p. 5) su empirismo es constructivo porque "concibe la actividad científica más como una construcción que como un descubrimiento: construcción de modelos que deben ser adecuados a los fenómenos, y no descubrimiento de la verdad respecto de lo inobservable". Según van Fraassen los modelos o idealizaciones no son inducidos a partir de la experiencia concreta sino que son creaciones, construcciones de un científico o una comunidad científica. Para él tampoco tiene sentido los intentos de los empiristas lógicos de reducir una teoría a términos observacionales exclusivamente; esto es una tarea imposible porque todo nuestro lenguaje es teórico.

Pasemos entonces a justificar la neutralidad de la concepción estructuralista de las teorías en relación a este problema de las entidades teóricas o inobservables y los términos que las nombran. En el contexto de su defensa del empirismo constructivo, van Fraassen (1989, p. 190) critica al programa estructuralista de asumir una posición instrumentalista porque él considera que este programa "insiste en que una 
teoría no es equiparable con el tipo de cosa de la cual se pueda decir propiamente que es verdadera o falsa". Considero que esta interpretación es incorrecta. Es cierto que, como vimos en el segundo apartado, para los estructuralistas una teoría hay que mínimamente identificarla con el elemento teórico $T=\langle K, I\rangle$ compuesto por el núcleo teórico $K$ y las aplicaciones propuestas $I$, de modo que una teoría sería por completo una estructura (estructura matemática), el tipo de cosa que no es susceptible de ser verdadera o falsa. Muy seguramente esta idea es la que ha llevado a van Fraassen a calificar a los estructuralistas de instrumentalistas, pero lo cierto es que van Fraassen no tiene en cuenta que los estructuralistas introducen la noción de aserción empírica como una relación entre las aplicaciones propuestas y el núcleo teórico, y este tipo de entidad, que es un enunciado, sí es susceptible de ser verdadera o falsa.

Veamos, entonces, más en detalle la forma como los estructuralistas resuelven el problema de la aplicación de la verdad a las teorías empíricas. Esta exposición también permite observar cómo aparecen las aplicaciones propuestas en la aserción empírica de una teoría, un punto clave en la argumentación que se presentará en el siguiente numeral. Desde el punto de vista estructuralista, toda teoría expresada en términos modelo-teóricos tiene asociada una aserción empírica que puede precisarse bastante bien mediante la herramienta de la teoría de conjuntos, definiendo primero el contenido teórico y después, a partir de este último, el contenido empírico de una teoría. Aquí dejaremos de lado esos desarrollos formales para centrarnos mejor en los aspectos relacionados con el contenido de la aserción empírica. Para ello recojamos la definición de aserción empírica de los estructuralistas: "Si $T=\langle K, I\rangle$ es un elemento teórico, entonces la aserción empírica idealizada de $T$ es $I \in C_{n}(K)$ " (Ibíd., p. 91).

Ahora bien, el contenido empírico $C_{n}(T)$ de la teoría $T^{9}$ son las consecuencias empíricas de la teoría, es decir, el efecto a nivel empírico de las restricciones de la parte formal $K$ de $T$. Pero, como es obvio, este efecto conjunto a nivel empírico debe estar expresado mediante conceptos $T$-no-teóricos únicamente. Es decir, el contenido empírico $C_{n}$ resulta de aquellos modelos parciales que son susceptibles de ampliarse mediante conceptos $T$-teóricos y cumplen las restricciones contenidas en las leyes, ligaduras y vínculos. De manera más concreta, una estructura hace parte del contenido empírico de una teoría si, estando descrita en términos no-teóricos respecto a la teoría, es posible ampliarla con conceptos teóricos de tal manera que cumpla las restricciones de la teoría.

¿Qué hemos de entender, entonces, por la aserción empírica, $I \in C_{n}(T)$ ? La aserción empírica plantea que el dominio de aplicaciones propuestas hace parte de, es un elemento de, el contenido empírico de la teoría. Aquí es secundario que la

\footnotetext{
${ }^{9}$ Que es equivalente a $C_{n}(K)$.
} 
relación sea de pertenencia y no de contenencia; es de pertenencia debido a que el contenido empírico es un conjunto de conjuntos, de modo que la aserción empírica plantea que uno de esos conjuntos del contenido empírico es el conjunto de aplicaciones. Lo importante aquí es que, para que se dé dicha relación de pertenencia, las aplicaciones propuestas deben estar descritas mediante conceptos no-teóricos respecto a la teoría en cuestión, del mismo modo en que lo están las estructuras del contenido empírico. En términos más concretos, la aserción empírica plantea que para todo $i \in I$, dado que $i$ es un modelo potencial parcial, $i$ se puede expandir mediante conceptos $T$-teóricos a un modelo potencial que cumple las leyes, ligaduras y vínculos de $T$. Por tanto, la aserción empírica afirma que los principios de la teoría pueden aplicarse correctamente a los sistemas empíricos pertenecientes a $I$. Finalmente, como puede observarse, la aserción empírica es susceptible de ser verdadera o falsa.

A partir de esta incorrecta interpretación de van Fraassen, la lección particular que hay que sacar es que, en el análisis de la estructura de las teorías, desde el punto de vista estructuralista los elementos centrales de una teoría no son dos (el núcleo y las aplicaciones, como supone van Fraassen) sino tres: núcleo, aplicaciones propuestas y aserción empírica. Del mismo modo, aunque van Fraassen insiste en que los elementos centrales de una teoría son los modelos teóricos y las hipótesis teóricas (la conocida formulación de Giere), en realidad en su propuesta también son tres los elementos clave: modelos, hipótesis teóricas y fenómenos. La clase de fenómenos es un elemento implícito en los otros dos, ya que una hipótesis teórica lo que hace es relacionar un fenómeno con un modelo, tal y como hemos visto más arriba ${ }^{10}$.

Aún más, las críticas semejantes a la de van Fraassen cometen otro error al pretender equiparar las reflexiones sobre la naturaleza y estructuras de las teorías con las reflexiones epistemológicas acerca de qué dice una teoría sobre el mundo. Si bien las dos cuestiones están relacionadas, es necesario distinguirlas; incluso es necesario distinguir estas dos inquietudes de una tercera sobre la forma como se caracteriza la experiencia y el papel que ésta tiene en la justificación de las teorías científicas, tal y como veremos a continuación. Respecto al primer punto, el mismo van Fraassen defiende que el análisis de la estructura general de las teorías científicas (ya sea desde la perspectiva sintáctica o semántica) es independiente, neutral, del debate epistemológico entre realistas y empiristas. Así que si los estudios estructuralistas tienen que ver exclusivamente con la naturaleza de las teorías, tal y como estoy proponiendo, no habría ninguna razón para pretender involucrar dicho programa en el problema de las entidades teóricas.

10 Estas ideas sobre los elementos determinantes en la estructura de una teoría, desde las perspectivas de van Fraassen y los estructuralistas, las he desarrollado y sustentado en Guerrero (2003) y Guerrero (2008). 
En concreto, considero que el programa estructuralista es neutral en el debate realismo/antirrealismo. Esta posición también la respalda Diederich (1996, p. 18): "la principal diferencia entre el empirismo constructivo y el estructuralismo es epistemológica. Mientras el estructuralismo es básicamente neutral en este respecto (aunque a una aserción de una teoría también la llame, notoriamente, su aserción empírica), van Fraassen insiste en una noción absoluta de observabilidad (de entidades y no de términos)". De modo que, dado que el estructuralismo se concentra en el análisis de la estructura de las teorías, no es correcto calificarlo de instrumentalista porque supuestamente (como afirma van Fraassen) no involucra en la identidad de una teoría un elemento que sea susceptible de ser verdadero o falso, así como tampoco es correcto calificarlo de no-instrumentalista por involucrar la aserción empírica asociada a un elemento teórico. Esto último es incorrecto porque la aserción empírica, bajo el espíritu estructuralista, sólo afirma una relación general entre el conjunto de aplicaciones propuestas y el núcleo teórico, una relación de carácter tan general como la que buscan van Fraassen y Giere con la introducción de las hipótesis teóricas en la identidad de una teoría, la cual no los compromete epistemológicamente. La aserción empírica en el estructuralismo no adelanta ningún tipo de compromiso respecto a las entidades inobservables supuestas por las teorías, pues ésta simplemente plantea que las aplicaciones propuestas están dadas en términos no-teóricos respecto a la teoría en cuestión, de tal manera que éstas pueden expandirse mediante conceptos teóricos a un modelo potencial que cumple las leyes, las ligaduras y los vínculos de la teoría. Espero que esta explicación aclare la duda expresada por Diederich al decir que "aunque a una aserción de una teoría también la llame, notoriamente, su aserción empírica".

Ahora bien, aunque el estructuralismo es neutral en el problema de las entidades inobservables o en el debate realismo/empirismo, tal y como espero haber justificado arriba, no lo es en el problema epistemológico de la fundamentación de las teorías, tal y como trato de argumentar a continuación.

\section{El coherentismo estructuralista en la fundamentación de las teorías}

Veamos entonces cómo se deriva el coherentismo del hecho de caracterizar las aplicaciones propuestas en términos únicamente de conceptos relativamente no-teóricos. Para ello se seguirá la estrategia metodológica del numeral anterior de contrastar los planteamientos estructuralistas con los de van Fraassen.

En el último apartado de An Architectonic for Science, "VIII. 6 Foundationalism versus coherentism", Balzer, Moulines y Sneed emprenden el estudio sobre la fundamentación de la ciencia y analizan las distintas posiciones filosóficas posibles al respecto, para finalmente concluir que el estructuralismo como programa filosófico 
es neutral en este asunto (véase página 423). Diederich (1996, p. 17. Lo del paréntesis es mío) también estaría apoyando esta idea de que el programa estructuralista es neutral en la fundamentación de las teorías (y no sólo en el debate realismo/antirrealismo) al considerar que la dicotomía estructuralista teórico/no-teórico no conlleva ningún compromiso epistémico, aunque extrañamente al final afirme que "el estructuralismo tiende a ser empirista":

Esta concepción [el empirismo constructivo de van Fraassen] comprende una dicotomía de entidades teóricas (no-observables) y observables que es análoga a la dicotomía teórico/no-teórico de Sneed. Esto lo compromete [a van Fraassen] con una posición antirrealista (agnóstica) respecto a las entidades teóricas y, al mismo tiempo, con una posición empirista respecto a las entidades observables. Esas dos posiciones no tienen paralelo en el estructuralismo como tal; es decir, el estructuralismo en sí mismo es neutral epistemológicamente, aunque el estructuralismo tiende a ser empirista.

Pues bien, lo que aquí se defiende es que, contrariamente a Diederich, tanto la dicotomía de van Fraassen como la estructuralista conllevan compromisos epistémicos: la primera, compartida por realistas y antirrealistas, conlleva a una especie de empirismo moderado; y la segunda a un coherentismo, tal y como se ha insistido, de tal manera que no es cierto que "el estructuralismo tiende a ser empirista", pues en realidad al derivar en un coherentismo niega cualquier forma de empirismo en la justificación de las teorías. En otras palabras, en los términos en los que van Fraassen plantea la discusión entre realistas y antirrealistas, tal y como se aclaró más arriba, hay que admitir tres cosas: una, que las dos perspectivas comparten el punto de partida de la distinción entre observables e inobservables; dos, que las perspectivas discrepan en cuanto a la existencia o no de inobservables; $y$, tres, que ambas perspectivas comparten la tesis de que la experiencia descrita en términos de observables constituye el tribunal último de las teorías científicas. Además, las dos últimas tesis son apuestas epistemológicas: la primera de éstas divide a los realistas de los antirrealistas (bajo la perspectiva de van Fraassen, insisto) y la última divide a realistas y antirrealistas de los estructuralistas, tal y como subraya Diederich, de tal manera que si hay un compromiso epistemológico en los primeros lo hay también en los segundos.

A continuación presento una reconstrucción bastante simplificada ${ }^{11}$ del análisis que hacen, en An Architectonic for Science, Balzer, Moulines y Sneed sobre las distintas posibilidades respecto a la justificación del conocimiento científico con el propósito de mostrar, contrariamente a ellos, que por este camino las tesis estructuralistas conducen a un coherentismo. Veremos que dicho análisis tiene en cuenta como alternativas el fundamentismo (extremo), el apriorismo y el coherentismo.

11 En Guerrero (2003), pp. 214-237, hago una reconstrucción más próxima a la original. 
Veamos cómo se presenta la justificación de una teoría en un caso concreto, aunque bastante simplificado. Partamos de una teoría $T_{0}$ que desde un punto de vista estructuralista calificamos de exitosa; de manera más particular, y para simplificar, digamos que $T_{0}$ tiene éxito al aplicarse a un determinado sistema empírico (fenómeno, experiencia), esto es, a una aplicación propuesta $i_{0}$ particular. De modo que la cuestión que nos interesa estudiar es la de en qué se fundamenta el éxito de $T_{0}$ o conocer el mecanismo que nos permite justificar el éxito de $T_{0}$.

En primer lugar, la respuesta a esta cuestión desde una posición fundamentista (extrema) estaría representada por los positivistas lógicos para quienes la experiencia u observación directa garantiza una base neutral para la evaluación de las teorías. Dos científicos pertenecientes a tradiciones científicas distintas no tendrían dificultades para ponerse de acuerdo en si una teoría da cuenta o no de determinada experiencia empírica. Así, en el caso particular de $T_{0}, T_{0}$ es una teoría exitosa porque $i_{0}$ representa una experiencia o fenómeno directamente observable que no ofrece ninguna duda y que, por tanto, nos proporciona un conocimiento seguro de la naturaleza; $\mathrm{y}$, además, $T_{0}$ da cuenta de esta experiencia porque sus predicciones en este punto, a partir principalmente de sus leyes, concuerdan con $i_{0}$. En síntesis, para el fundamentista (extremo) la experiencia, entendida de esta forma extrema, es la piedra de toque de las teorías empíricas.

La objeción estructuralista a este planteamiento recoge algunos elementos de la crítica que hicieron los filósofos historicistas (Hanson, Kuhn y Feyerabend, entre otros) en su momento a esta particular manera como entendieron los positivistas lógicos la experiencia. De acuerdo con aquéllos, no hay tal experiencia neutral, independiente de una teoría; por el contrario, la observación está cargada de teoría, toda experiencia requiere ser conceptualizada desde algún marco teórico o teoría, de modo que dos científicos que trabajan en teorías distintas y que sean expuestos a lo mismo (con experiencias perceptivas cualitativamente idénticas), darían informes de observación distintos. Pero estructuralistas e historicistas no coinciden completamente en la forma de entender la experiencia, los últimos son más radicales. Como podemos ver en la cita anterior y por la manera particular como comprenden las aplicaciones propuestas, los estructuralistas enfatizan que toda experiencia ha de ser conceptualizada desde una teoría (no hay observaciones teóricamente neutras) y en que una aplicación particular, digamos $i_{0}$, de una teoría $T_{0}$ tiene que estar conceptualizada en términos de una teoría precedente, digamos $T_{1}$. Stegmüller (1996, p. 18. Lo del paréntesis es mío) expresa muy bien la diferencia en estos términos: hay que distinguir entre la tesis de la carga teórica de las constataciones de hechos y la tesis de la relativización de los términos teóricos a una teoría. La diferencia radica en que en la tesis estructuralista "lo que es un hecho [experiential data] para una teoría viene determinado por otra teoría (iy no por esa teoría misma) [como sí 
sucede en la tesis de la carga teórica]". Por tanto, tomemos nota de la diferencia y de que ambas tesis coinciden en que lo que es un hecho viene determinado teóricamente, depende de una teoría.

Bajo este último presupuesto, pasemos entonces a examinar las otras alternativas en la fundamentación de la teoría $T_{0}$. Puesto que $T_{0}$ tiene éxito al aplicarse a $i_{0}$, podemos admitir que $T_{0}$ da cuenta de $i_{0}$ en el sentido en que $i_{0}$ puede expandirse a un modelo potencial que cumple las leyes de $T_{0}$ (es decir, a un modelo actual) mediante la introducción de términos $T_{0}$-teóricos apropiados, pero por el momento no tenemos garantizado un conocimiento seguro en relación con $i_{0}$, un conocimiento que tenga una certeza semejante a la propuesta por el fundamentismo (extremo) a través de la experiencia directa y neutral. No se tiene este tipo de garantía, plantea el estructuralismo, precisamente porque la descripción de $i_{0}$ depende de otra teoría distinta a $T_{0}$. Por tanto, con el propósito de justificar el éxito de $T_{0}$, desde una perspectiva estructuralista, no es suficiente con decir que $T_{0}$ da cuenta de $i_{0}$ sino que es necesario hacer explícito que esto es así teniendo presente que $i_{0}$ está descrita mediante términos $T_{0}$-no-teóricos o, de manera más exacta, con términos que son teóricos respecto a otra teoría (digamos $T_{1}$ ) subyacente a $T_{0}$, de tal manera que la determinación de $i_{0}$ se hace a través de las leyes de $T_{1}$. Así, pues, en busca de una justificación de $T_{0}$ encontramos que ésta depende de otra teoría $T_{1}$, aunque en un primer momento parecía que ésta dependía sólo de $i_{0}$.

Ahora bien, vistas así las cosas, parece que aquí nos encontramos en una regresión infinita o en un círculo vicioso. Entraríamos en una regresión infinita si con el propósito de justificar el éxito de $T_{0}$ nos vemos forzados a justificar el éxito de $T_{1}$, y para justificar el de esta última tenemos que recurrir a justificar el de una tercera teoría $T_{2}$, y así sucesivamente. En otras palabras, como antes, para justificar el éxito de $T_{1}$ podemos partir de que $T_{1}$ tiene éxito al aplicarse a la aplicación propuesta $i_{1}$ y suponer además que en la descripción de $i_{1}$ sólo empleamos términos que son $T_{1^{-}}$no-teóricos y, digamos entonces que son $T_{2}$-teóricos. De modo que la fundamentación de $T_{0}$ resulta dependiendo de $T_{2}$ porque la fundamentación de $T_{1}$ depende de $T_{2}$ y así sucesivamente. Finalmente no tendríamos justificación para $T_{0}$, lo cual sería inaceptable.

Enfrentaríamos un círculo vicioso si ante la necesidad de justificar el éxito de $T_{1}$, ya que $T_{1}$ hace parte de los elementos que permiten justificar el éxito de $T_{0}$, recurrimos para ello a $T_{0}$ o a cualquier otra teoría cuya fundamentación en últimas dependa de $T_{0}$ o $T_{1}$. Desde luego que desde un principio excluimos estas dos posibilidades como explicaciones posibles del proceso de fundamentación de las teorías científicas puesto que nos proporcionan una imagen totalmente contradictoria y, por tanto, imposible de darse en la actividad científica. 
Pero aún quedan otras dos alternativas. Una que podemos calificar de fundamentismo apriorista y otra de coherentista, las cuales se obtienen eludiendo la regresión infinita y el círculo vicioso, respectivamente. El proceso de justificación del éxito de $T_{0}$ se puede dar por terminado en una teoría $T_{a}$ que tendría un carácter $a$ priori, esto es, que se autojustificaría independiente de cualquier otra teoría o conocimiento. Una primera objeción que se le puede hacer a esta alternativa es que para evitarnos tantas vueltas podríamos adjudicarle dicho carácter a priori a la misma teoría inicial $T_{0} \mathrm{o}$ a cualquier otra teoría anterior ya que no tenemos ningún criterio que nos permita distinguirlas entre sí en este sentido. Esta alternativa también es problemática a la hora de proporcionar una solución a la evaluación de las teorías porque no tendríamos criterios epistémicos objetivos que permitan justificar este carácter a priori, si acaso se podrían esgrimir criterios psicológicos, sociológicos o de cualquier otro tipo.

Finalmente, se obtendría una posición coherentista al hacer desaparecer lo vicioso del círculo vicioso, esto es, si en vez de pedir una justificación de $T_{0}$ mediante $T_{1}$ sólo se exige que $T_{0}$ sea coherente con $T_{1}$, dando por sentado que $T_{1}$ es coherente con el resto de teorías existentes. Más concretamente, en términos estructuralistas, que lo que dice $T_{0}$ sobre $i_{0}$ no es contradictorio con la descripción que hace $T_{1}$ de $i_{0}$ y $T_{1}$ es coherente con sus teorías subyacentes en este mismo sentido. Hay que aclarar que este tipo de coherentismo no es un coherentismo interno en el que se exige que la teoría no sea autocontradictoria. Esto se da por cumplido, de modo que de lo que aquí se trata es de un coherentismo global en el que la teoría no sea contradictoria con el resto de teorías con las cuales está relacionada.

Por tanto, concluyo, el estructuralismo desemboca en este coherentismo global respecto a la evaluación de las teorías empíricas porque de las distintas alternativas examinadas en la argumentación anterior ésta es la única plausible que es totalmente compatible con los presupuestos estructuralistas. En síntesis, considero que este coherentismo se debe a que una de las ideas que promueve la concepción estructuralista en su concepto de aplicación propuesta es la dependencia teórica de la experiencia (tesis que no es equivalente a la tesis de la carga teórica de la observación): las descripciones de las aplicaciones propuestas es una cuestión más bien de relaciones entre teorías que de relaciones entre éstas y el mundo; los informes observacionales más que proporcionar información sobre el mundo, presentan relaciones entre teorías; y la cuestión no es sólo que la experiencia sea conceptualizada, sino que no es posible entenderla de un modo objetivo, independientemente de un esquema conceptual.

Por tanto, los estructuralistas se alejan en este punto de una posición empirista moderada. Un empirismo moderado no estaría de acuerdo con la idea de que, desde una teoría dada, calificar la descripción de una aplicación propuesta de correcta o incorrecta está supeditado a una segunda teoría; para éste el que una aplicación sea 
correcta o incorrecta tiene que ver directamente con la experiencia, no con una teoría. Además, parece que es posible decir sin contradecirse que, por una parte, los informes de observación obtenidos de la actividad experimental son informativos acerca del mundo y, en este sentido, independientes de cualquier teoría; y, por la otra, que esos informes son al mismo tiempo dependientes de un lenguaje, de un esquema conceptual, en el sentido de que su formulación se hace con un lenguaje y dentro de un esquema de conceptos proporcionados de antemano o construidos para tal fin.

En otros términos, considero que el coherentismo es una alternativa inadecuada porque contradice aquella intuición (¿empirista?) de que la experiencia es importante en la valoración de las teorías empíricas, de ahí que califiquemos a este tipo de teorías como empíricas, véase al respecto Rosenberg (2005, p. 89). En realidad hay un empirismo moderado con las características mencionadas arriba, que se dejó por fuera en el análisis anterior y es una alternativa distinta al fundamentismo (extremo), al apriorismo y al coherentismo. Esta alternativa es una especie de fundamentismo moderado y corresponde al empirismo posfundamentista que desarrolla van Fraassen "en contraste con aquellas variedades de empirismo identificadas como el último bastión del fundamentismo en epistemología" (van Fraassen, 1993, p. 6)12. Este empirismo recoge positivamente el rechazo del fundamentismo extremo, esto es, en palabras de van Fraassen, "el Mito de lo Dado. No hay juicios que sean neutros teóricamente, epistémicamente seguros y autojustificables. No hay fundamentos de la creencia racional" (van Fraassen, 1993, p. 7).

Esto es, en términos negativos, dos ideas en concreto son las que defiende este empirismo: la imposibilidad de obtener una fundamentación absoluta y segura del conocimiento científico por cualquier medio, incluida la experiencia, y la inexistencia de una observación descontaminada de cualquier teoría. En términos positivos: si bien la descripción de las experiencias (tanto ordinarias como científicas) hay que hacerlas mediante la implementación de un lenguaje particular (la contraparte correspondiente a la segunda idea), la experiencia constituye el medio para fundamentar el conocimiento, aunque no sea en forma absoluta y segura (la contraparte correspondiente a la primera idea). El hecho de que los informes experimentales y

\footnotetext{
12 Aquí surge la inquietud de si este empirismo es equivalente al empirismo constructivo desarrollado por el mismo van Fraassen. En el mismo artículo, van Fraassen presenta este empirismo como una "filosofía de la ciencia antirrealista" (palabras suyas), una alternativa opuesta al realismo y al relativismo. Pero considero que tal equivalencia es incorrecta, pues en realidad este empirismo posfundamentista que precisa qué ha de entenderse por experiencia, esto es la teoría pragmática de la observación defendida por van Fraassen, también haría parte del realismo. El mismo van Fraassen reconoce esto, al comienzo del artículo, cuando dice: "me pareció que esta versión de la observación era una parte central del realismo científico, así que la hice participar de mi oposición" (p. 14). Rosenberg (2005, p. 97) también apoya ésta interpretación que estoy presentando.
} 
de observación de los científicos sean presentados mediante un lenguaje particular que ha sido moldeado históricamente a través de teorías y otros medios más, no implica que con un cambio de lenguaje (o de nuestras creencias sobre el mundo) también cambie lo que es observable en el mundo. El hecho de que sólo podamos hablar del mundo desde un lenguaje o un esquema conceptual o algo por el estilo, no puede llevarnos a borrar la distinción entre lenguaje y mundo; una ilustración de van Fraassen: "nuestra opinión sobre la cantidad de agua presente en Marte tampoco es constante a lo largo de la historia de la ciencia. Sin embargo, la masa de agua en Marte no ha estado cambiando junto con este cambio de opinión" (van Fraassen, 1993, p. 20).

De modo que la lectura que hace van Fraassen del rechazo de las tesis del fundamentismo (extremo) no lo lleva a posiciones extremas en donde se abandone la fundamentación epistémica de las teorías científicas en la experiencia, como sí sucede con la tesis de la dependencia teórica de la experiencia contenida en el estructuralismo y con el relativismo propio de la tesis de la carga teórica de la observación, que sería una tesis más fuerte que la estructuralista.

En síntesis, hemos visto cómo el hecho de caracterizar las aplicaciones propuestas de una teoría en términos de conceptos no-teóricos respecto a la teoría, implica que la experiencia es dependiente teóricamente, lo cual conduce al programa estructuralista a un coherentismo en la fundamentación de las teorías empíricas. Aunque la tesis estructuralista de la dependencia teórica de la experiencia es más débil que la versión extrema de la tesis de la carga teórica de la observación que conduce al relativismo. El coherentismo es inaceptable porque contradice aquella idea de que la experiencia (independiente teóricamente) interviene de un modo importante en la valoración de las teorías empíricas. Por tanto, se ha presentado, sin mayores desarrollos, una alternativa empirista moderada en el tema de la fundamentación de las teorías.

\section{Referencias bibliográficas}

Balzer, W. y Moulines, C. U. (Eds.) (1996): Structuralist Theory of Science, Berlin, Walter de Gruyter.

Balzer, W. y Moulines, C. U. (2000): "Introduction", en Balzer, W.; Moulines, C. U. y Sneed, J. D. (Ed.), Structuralist Knowledge Representation, Amsterdam, Rodopi, pp. 5-17.

Balzer, W.; Moulines, C. U.; y Sneed, J. D. (1987), An Architectonic for Science. The Structuralist Program, Dordrecht, Reidel.

Balzer, W.; Moulines, C. U.; y Sneed, J. D. (Eds.) (2000): Structuralist Knowledge Representation, Amsterdam, Rodopi. 
DieDERICH, W. (1996): "Structuralism as Developed Within the Model-Theoretical Approach in the Phlosophy of Science", en Balzer, W. y Moulines, C. U. (Eds.) (1996), pp. 15-22.

DíEz, J. A. y Moulines, C. U. (1997): Fundamentos de filosofia de la ciencia, Barcelona, Ariel.

Guerrero, G. (2003): Enfoque semántico de las teorías. Estructuralismo y espacio de estados: coincidencias y divergencias, Tesis doctoral, Madrid, Universidad Complutense de Madrid, (Publicación en cd-rom).

Guerrero, G. (2008): "Individuación de las teorías en el enfoque semántico", Principia 12/1, pp. 97-119.

Moulines, C. U. (1996): "Structuralism: The Basic Ideas", en Balzer, W. y Moulines, C. U. (Eds.) (1996), pp. 1-14.

Moulines, C. U. (2002): "Introduction: Structuralism as a Program for Modelling Theoretical Science", Synthese volume 130/No.1, pp. 1-11.

Nagel, E.; Suppes, P. y Tarski, A. (Eds.) (1962): Logic, Methodology, and Philosophy of science: Proceedings of 1960 International Congress, Stanford, Stanford University Press.

Putnam, H. (1962): "What theories are not", en Nagel, E.; Suppes, P. y Tarski, A. (Eds.) (1962).

Rivadulla, A. (1985): "Las concepciones realista y estructuralista del progreso científico", Teorema XV/1-2, 1985, pp. 245-257.

Rosenberg, A. (2005): Philosophy of Science, New York, Routledge.

SneEd, J. (1971): Logical Structure of Mathematical Physics, Dordrecht, Reidel.

StegmülleR, W. (1976): The Structure and Dynamics of Theories, New York, Springer-Verlag.

VAN FraAsSEN, B. C. (1970): "On the extension of Beth's semantics of physical theories", Philosophy of Science, pp. 325-339.

Van Fraassen, B. C. (1980): The Scientific Image, Oxford, Clarendon Press; v.e. La imagen cientifica, México, Paidós-UNAM, 1996.

VAn FraAssen, B. C. (1989): Laws and Symmetry, Oxford, Clarendon Press.

Van FraAssen, B. C. (1993): "From Vicious Circle to Infinite Regress, and Back Again", PSA vol. 2, pp. 6-29.

Germán Guerrero Pino

Departamento de Filosofía

Universidad del Valle

Cali - Colombia

germangpino@gmail.com 